\title{
Comunicación
}

\section{Conducta del Puma Andino Puma concolor (Linnaeus, 1771) en cautiverio Bajo un Programa de Enriquecimiento Ambiental en el Parque Zoológico «Taraccasa» (Apurímac, Perú)}

\author{
Behavior of Andean Puma Puma concolor (Linnaeus, 1771) in Captivity Under an \\ Environmental Enrichment Programme in the Zoo «Taraccasa» (Apurímac, Peru) \\ Cristian J. Morales Mijahuanca ${ }^{1}$, Roger Machaca M. ${ }^{2}$, Edgar Quispe Peña ${ }^{3}$, \\ Víctor Cano Fuentes ${ }^{4}$, Max H. Escobedo Enriquez ${ }^{4}$, Flor A. Corredor ${ }^{4}$, \\ Virgilio Machaca Machaca ${ }^{4,5}$
}

\section{Resumen}

\begin{abstract}
Se aplicó un programa de enriquecimiento ambiental a pumas (Pumas concolor) del Parque Zoológico Taraccasa en Abancay (Apurímac, Perú) con el objetivo de monitorear cambios de conducta. Se observaron cuatro pumas (tres hembras, un macho) de 3 a 9 años de edad. Se utilizaron cuatro tipos de enriquecimiento ambiental: físico (taburetes de madera, troncos), alimenticio (carne de equino, alimento balanceado de gatos), sensorial (costales con esencias aromáticas y especias, orines de zorros y venados), y social (pelotas con piel de equino). El estudio consideró la etapa previa al enriquecimiento ambiental (24 días) y la etapa durante el enriquecimiento ambiental (24 días). Se registraron las conductas entre 09:00 a 12:00 y entre 16:00 a 18:00 con cámara de video. Las conductas individuales con incremento significativo fueron el cuidado corporal, locomoción, observación, exploración, búsqueda $(\mathrm{p}<0.001)$ y disminución de un comportamiento anormal como el pacing $(\mathrm{p}<0.001)$, mientras que en las conductas grupales se incrementaron la aproximación y el marcaje $(\mathrm{p}<0.001)$ y disminuyó la persecución $(\mathrm{p}<0.001)$. Se concluye que el programa de enriquecimiento ambiental aplicado a los pumas tuvo un efecto positivo, al aumentar los comportamientos de actividad y sociales normales y disminuir la estereotipia.
\end{abstract}

Palabras clave: Puma concolor; enriquecimiento ambiental; estereotipias; bienestar animal

\footnotetext{
${ }^{1}$ Municipalidad Distrital de Huancarama, Apurímac, Perú

${ }^{2}$ Instituto Superior Tecnológico Público de Sangara Sicuani, Cusco, Perú

${ }^{3}$ Vicepresidencia de Investigación, Universidad Nacional Autónoma de Chota, Cajamarca, Perú

${ }^{4}$ Universidad Nacional Micela Bastidas de Apurímac, Facultad de Medicina Veterinaria y Zootecnia, Abancay, Apurímac, Perú

${ }^{5}$ E-mail: machacav@hotmail.com
}

Recibido: 10 de diciembre de 2016

Aceptado para publicación: 14 de junio de 2017 
An environmental enrichment programme was applied to pumas (Pumas concolor) of the Taraccasa Zoological Park in Abancay (Apurímac, Peru) to monitor behavioural changes. Four pumas or cougars (three females, one male) from 3 to 9 years old were observed. Four types of environmental enrichment were used: physical (wooden stools, tree trunks), food (equine meat, balanced feed for cats), sensorial (sacks with aromatic essences and spices, urine of foxes and deer), and social (balls made with equine skin). The study considered the stage prior to environmental enrichment ( 24 days) and the stage during environmental enrichment ( 24 days). The behaviours were recorded with video camera between 09:00 to 12:00 and between 16:00 to 18:00. Individual behaviours with a significant increase were body care, locomotion, observation, exploration, search $(p<0.001)$ and reduction of abnormal behaviour such as pacing $(p<0.001)$ was also observed, while group behaviours that increased were approximation and marking $(p<0.001)$, and chasing decreased $(p<0.001)$. It is concluded that the environmental enrichment programme applied to the pumas had a positive effect, increasing normal activity and social behaviours, and reducing stereotypy.

Key words: Puma concolor; environmental enrichment; stereotypes; animal welfare

\section{INTRODUCCIÓN}

Los animales silvestres disponen del tiempo y energía para buscar alimentos, construir nidos, defender su territorio y buscar pareja para aparearse. En cautiverio, el manejo cuidadoso de los animales y el fácil acceso a los alimentos, refugios y pareja reducen significativamente las actividades y el tiempo destinado a ellas (Beresca, 2014). Un ambiente de cautiverio usualmente presenta condiciones para las cuales todas las especies deben adaptarse, como la presencia humana, imposición de regímenes de alimentación y una falta de oportunidades de búsqueda de alimento, así como a los procedimientos médicos veterinarios, las limitaciones de espacio, la agrupación social impuesta y el entorno inmutable (Broom, 1988; Morgan, 2006).

El enriquecimiento ambiental es un principio de manejo animal que busca mejorar la calidad del cuidado de los animales en cautiverio, identificando y proporcionando los estímulos ambientales necesarios para un óptimo bienestar psicológico y fisiológico
(Newberry, 1995). El Puma concolor es un animal de comportamiento nocturno en estado silvestre (Rodríguez et al., 2006). Se han hecho numerosos intentos en Latinoamérica para aumentar el bienestar de los pumas en cautiverio, enriqueciendo los ambientes en los que se mantienen, obteniéndose un incremento de conductas de actividad normales de la especie (Pacheco, 2005; Medrano, 2008; Villafuerte, 2012; Martínez-Pilido et al., 2014).

Comportamientos normales (independientemente que fueran individuales o sociales) corresponden al descanso, el paseo, al acicalamiento y a trepar. Las estereotipias más frecuentes en felinos son paseos cortos y desesperados, denominado en inglés como pacing, así como letargia (Mason y Rushen, 2006; Villafuerte, 2012).

El Parque Zoológico Taraccasa, en Abancay, Perú, cuenta con 52 animales en exhibición. Una de las zonas del establecimiento cuenta con dos ambientes destinados a los pumas (Puma concolor). Estos animales presentan las conductas estereotípicas de pacing y acicalamiento excesivo; sin embar- 
go, este zoológico no cuenta con programas de enriquecimiento ambiental. El objetivo del presente estudio fue evaluar la influencia del enriquecimiento ambiental sobre las conductas en el puma andino del Parque Zoológico «Taraccasa».

\section{Materiales y Métodos}

\section{Animales e Instalaciones}

El Parque Zoológico Taraccasa, institución dependiente de la Municipalidad Provincial de Abancay, Perú, se localiza entre las coordenadas N8493141 - E731311, a una altitud de $2828 \mathrm{msnm}$, y presenta una temperatura media anual de $25.9^{\circ} \mathrm{C}$.

Los pumas son exhibidos de lunes a domingo, durante todo el día, ya que el zoológico no cuenta con cerco perimétrico. En cada recinto habitan dos pumas (Cuadro 1). El recinto uno tiene un área de $842.4 \mathrm{~m}^{2} \mathrm{y}$ el recinto dos de $828.0 \mathrm{~m}^{2}$. Los dos ambientes tienen características básicas similares, con paredes de malla de cocos de acero inoxidable, piso de pasto y tierra, con diferentes niveles de inclinación (30\% en promedio). El ambiente uno presenta un cubil y el ambiente dos no lo tiene. La alimentación consistía en $2 \mathrm{~kg}$ de carne de caballo, 6 veces por semana y 1 día de ayuno. Los martes recibían $200 \mathrm{~g}$ de hígado de equino. El estudio se realizó entre julio y setiembre de 2014, cuando no había lluvias.

\section{Características del Estudio}

El estudio tuvo una duración de 48 días (240 h de observación), distribuidos en dos fases: a) En la primera fase (antes del enriquecimiento ambiental) se realizó una observación de 24 días $(120 \mathrm{~h})$; siendo 5 h (300 min) el tiempo total de observación por día, $y$ b) en la segunda fase (durante el enriquecimiento ambiental) se realizó una observación de 24 días ( $120 \mathrm{~h})$, siendo igualmente $5 \mathrm{~h}$ (300 min) el tiempo total de observación por día.
El intervalo entre las dos fases de investigación fue de 14 días, en donde se acondicionó el recinto de los pumas y donde hubo un periodo de acostumbramiento de los mismos al ambiente enriquecido.

Se utilizó la técnica de muestreo focal. Se registraron las conductas durante 48 días, de lunes a domingo, por las mañanas de 09:00 a 12:00 y por la tarde de 16:00 a 18:00 horas. Estos horarios fueron considerados con base a observaciones previas donde los pumas permaneces inactivos temprano en las mañanas y entre 12:00 y 16:00 h. Se emplearon cuatro cámaras para grabar las conductas realizadas, sin que el investigador intervenga en el horario de grabación para no influenciar la conducta de los pumas. Los comportamientos fueron analizados en el gabinete al observar los videos.

\section{Enriquecimiento Ambiental}

Se utilizaron los cuatro tipos de enriquecimiento ambiental:

Físico

Se podó el pasto y se retiraron las malezas, piedras y huesos. Se armó un taburete de madera de eucalipto de $2 \mathrm{~m}$ de alto con una plataforma de $3 \mathrm{~m}^{2}$ en el recinto uno ( $\mathrm{Fi}$ gura 1) y un taburete de $2 \mathrm{~m}$ de altura con una plataforma de metal de $6 \mathrm{~m}^{2}$ en el recinto dos. Además, se colocaron palos de eucalipto en forma vertical de $2.5 \mathrm{~m}$ en ambos recintos. En el ambiente uno se construyó una estructura con techo de calamina para proporcionar sombra.

\section{Alimentación}

Las raciones de carne e hígado fueron ofrecidas en varias presentaciones:

- Dentro de costales de azúcar, atado con soga de cabuya, amarrado a los palos verticales.

- Carne en costales de azúcar con paja, colgados en los palos verticales. 


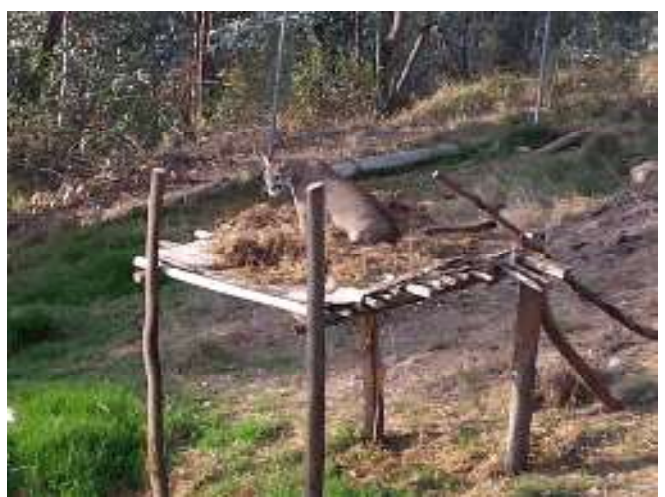

Figura 1. Puma sobre el taburete en busca de sus alimentos

- Costales de azúcar con paja y aromas de menta, donde se optó a llenar algunos costales con carne. Todos los costales fueron vertidos con sangre de equino.

- Carne dispersa en todo el recinto, escondido con ramas, hojas y maderas.

- Carne escondida encima del taburete, cubierta por paja.

- Carne colgada en los troncos con soga cabuya.

- Carne dentro de maniquís de cartón, con forma de vicuña (Vicugna vicugna) y venado de cola blanca (Odocoelius virginianus).

- Carne dentro de cajas de cartón, atadas con soga de cabuya y colgada en los postes de madera.

- Croquetas para gato con sangre de equino, presentados en lugares visibles.

- Croquetas para gato disperso por el ambiente.

- Hígado disperso por el recinto.

- Hielos de sangre esparcido en el recinto y colgados en los troncos de madera.

\section{Estimulación sensorial}

Se emplearon diversos estímulos para captar la atención del olfato, gusto, tacto, visión y audición de los pumas:

- Olfativo: Se utilizaron esencias aromáticas como la menta y varias especias. Se le hizo hervir y, una vez fría, se esparció en varias partes de los recintos. Asimismo, se utilizó orines de Lycalopex culpaeus (zorro andino) y $O$. virginianus (venado de cola blanca) rociándolo por fuera del recinto.

- Gusto: Se varió el tipo de alimento, usando carne de equino, vísceras y croquetas de gato.

- Tacto: Se trabajó con varias texturas de suelo. Se agregó arena en los lugares donde no frecuentaban, pelotas de juguete hechas con piel de equino y cocidas con soga de cabuya; así como los costales con las variantes de alimentos ofrecidos. Además, se colocaron troncos que les sirvieron para afilar sus garras y para el marcaje territorial.

\section{Estimulación social y cognitiva}

Los tres estímulos antes mencionados conllevaron a un estímulo social, mientras que los enriquecimientos empleados ayudaron a desarrollar estímulos cognitivos.

\section{Comportamiento anormal}

Se registraron movimientos estereotipados como: caminar sobre el mismo sitio una y otra vez, actividad conocida con el término inglés de pacing (Estrada y Parra, 2007).

\section{Análisis Estadístico}

Se utilizó un modelo aditivo de un factor con medidas repetidas en el tiempo. Se tuvo como factor en estudio los periodos antes y durante el enriquecimiento ambiental.

\section{Resultados}

El análisis de las observaciones realizadas parece sugerir cambios en los niveles de actividad y comportamientos estereotípicos después del enriquecimiento ambiental. Se registraron 17 tipos de comportamientos (8 individuales y 9 grupales). Se identificaron dos 
Cuadro 1. Registro y edad de los pumas

\begin{tabular}{ccccc}
\hline Recinto & Nombre & Sexo & Edad (años) & Tipo de ingreso \\
\hline 1 & Princesa & Hembra & $9^{1}$ & Custodia temporal $^{2}$ \\
1 & Bryan & Macho & $9^{1}$ & Custodia temporal \\
2 & Bella & Hembra & 3 & Nacimiento \\
2 & Yenni & Hembra & 4 & Nacimiento \\
\hline
\end{tabular}

${ }^{1}$ Edad aproximada

${ }^{2}$ La custodia temporal fue otorgada por el Instituto Nacional de Recursos Naturales (INRENA)

Cuadro 2. Tiempo ${ }^{1}$ dedicado a conductas individuales y grupales de cuatro pumas (Puma oncolor) antes y durante el enriquecimiento ambiental de sus recintos en el Parque Zoológico Taraccasa (Abancay, Perú)

\begin{tabular}{lccc}
\hline \multirow{2}{*}{ Conductas } & Antes & Durante & Probabilidad $^{2}$ \\
\cline { 2 - 4 } & Promedio \pm d.e. & Promedio \pm d.e. & Periodo \\
\hline Individuales & & & \\
Cuidado corporal & $22.0 \pm 7.7$ & $28.2 \pm 9.6$ & $* *$ \\
Descanso & $132.6 \pm 24.2$ & $128.3 \pm 19.1$ & $\mathrm{NS}$ \\
Trófico & $33.9 \pm 4.8$ & $34.1 \pm 5.4$ & $\mathrm{NS}$ \\
Locomoción & $16.3 \pm 5.7$ & $22.2 \pm 6.4$ & $* *$ \\
Observación & $4.8 \pm 3.1$ & $10.2 \pm 4.5$ & $* *$ \\
Exploración & $0.8 \pm 0.8$ & $5.1 \pm 3.6$ & $* *$ \\
Búsqueda & $2.3 \pm 1.3$ & $9.6 \pm 7.7$ & $* *$ \\
Pacing & $57.8 \pm 7.8$ & $47.8 \pm 16.0$ & $* *$ \\
Grupales & & & \\
Juego & $0.8 \pm 1.7$ & $1.1 \pm 1.5$ & $\mathrm{NS}$ \\
Aproximación & $1.0 \pm 1.2$ & $1.9 \pm 2.2$ & $* *$ \\
Acicalamiento & $2.8 \pm 3.2$ & $2.5 \pm 2.9$ & $\mathrm{NS}$ \\
Persecución & $4.0 \pm 7.5$ & $1.2 \pm 1.5$ & $* *$ \\
Monta & $14.2 \pm 16.4$ & 0 & \\
Marcaje & $0.8 \pm 0.6$ & $3.0 \pm 3.3$ & $* *$ \\
Agresión & $3.0 \pm 2.0$ & $3.3 \pm 2.1$ & $\mathrm{NS}$ \\
Sumisión & $1.0 \pm 0$ & $1.2 \pm 0.4$ & \\
Vocalización & $4.2 \pm 5.2$ & $2.8 \pm 1.4$ & $\mathrm{NS}$ \\
\hline
\end{tabular}

${ }^{1}$ Tiempo dedicado durante 300 minutos de observación (5 horas por día) 
comportamientos estereotipados dentro de los comportamientos individuales: el pacing y el excesivo cuidado corporal.

Se halló una diferencia significativa en la proporción de comportamientos clasificados como actividad normal (Cuadro 1). El comportamiento individual con mayor tiempo de diferencia fue el de búsqueda entre la etapa sin enriquecimiento y la etapa con enriquecimiento ambiental (2.3 y $9.6 \mathrm{~min}$ ); asimismo, la observación (4.8 y $10.2 \mathrm{~min}$ ) y la exploración ( 0.8 y $5.1 \mathrm{~min})$ presentaron diferencia altamente significativa entre las dos etapas, incrementándose el tiempo de ejecución de estos comportamientos cuando se introdujeron los elementos enriquecedores.

En forma similar, en la conducta grupal el comportamiento con mayor tiempo de diferencia antes y después del enriquecimiento ambiental fue el de marcaje ( 0.8 y $3.0 \mathrm{~min}$, respectivamente), seguido de los comportamientos de aproximación ( 1.0 y $1.9 \mathrm{~min}$ ), y la conducta de persecución (4.0 y $1.2 \mathrm{~min})$, ocurriendo en este último caso una disminución de la actividad (Cuadro 2). No se tomó en cuenta la monta, ya que este comportamiento no fue influido por las variables en estudio.

La conducta estereotipada donde se observó una disminución de la actividad por el enriquecimiento ambiental fue el pacing $(47.8 \pm 16.0 \mathrm{~min}$ vs. $57.8 \pm 7.8 \mathrm{~min})$. Asimismo, el tiempo otorgado a la conducta de cuidado corporal se incrementó de $22.0 \pm 7.7$ $\min$ a $28.2 \pm 9.6 \min (\mathrm{p}<0.001)$.

\section{Discusión}

Los resultados del estudio indican que al dificultar el acceso a los alimentos, ya sea escondiéndolo o colgándolos, los pumas aumentan el tiempo de búsqueda, y de esa forma, aumentan otros comportamientos naturales como el caminar, saltar, olfatear y observar. El marcaje es una conducta que au- mentó significativamente cuando se colocaron palos de madera dentro del recinto, además, disponían de más lugares para afilar sus garras y lo hacían por más tiempo. Los olores de otros animales también ayudaron a aumentar este comportamiento, pues los pumas dejaban marcas en las fronteras de sus territorios para defenderlos de otros pumas.

En forma similar al estudio de Villafuerte (2012), luego de introducir elementos enriquecedores, se observó que las hembras pasaban más tiempo caminando, trepando, saltando y observando, mientras que el macho pasaba más tiempo echado. Díaz (2001) comenta que cuando se provee a los animales de estímulos y se cambian sus métodos de alimentación, se incrementa el tiempo de locomoción, como lo encontrado en los pumas de esta investigación. Uno de los comportamientos que no tuvo disminución significativa fue la conducta de descanso, a comparación de otros investigadores que encontraron diferencias con la introducción de elementos enriquecedores en el ambiente de los felinos (Broom, 1988; Medrano, 2008). La razón pudo deberse a que los felinos presentan mayor tiempo de actividad en las noches (Pacheco, 2005; Rodríguez et al., 2006; Harmsen et al., 2010).

Los pumas realizaron el pacing entre las 16 y 18 horas, cuando el sol ya no calentaba. Al introducirse elementos enriquecedores en la segunda etapa del estudio, los pumas sintieron curiosidad por estos, dándoles más tiempo a ellos y, por lo tanto, reduciendo el tiempo dedicado al pacing.

Medrano (2008) señala que se debe tener en cuenta que los felinos muestran una tendencia a incrementar estereotipias luego de haber pasado tiempo con los enriquecedores, como un resultado de frustración al no encontrar nuevos objetos después de terminar con los objetos introducidos. En el presente estudio, cuando la segunda etapa de investigación ya se encontraba avanzada, los elementos introducidos dentro del recinto ya 
eran parte normal del ambiente, y solo los elementos enriquecedores con comida, olores y sonidos era lo único que distraía a los pumas, perdiendo poco a poco el interés por ellos.

El cuidado corporal se presentaba luego que los pumas terminaran de consumir sus alimentos, tal como menciona Manteca (2003). Esta conducta participa en los mecanismos de termorregulación, ya que la saliva que se deposita en la superficie corporal contribuye a la pérdida de calor (Medrano, 2008; Mills et al., 2010). Si bien es cierto el cuidado corporal es una conducta normal y básica en los felinos, una conducta que excede el tiempo normal en que se realiza se puede convertir en una estereotipia (Mason y Rushen, 2006; Skibiel et al., 2007; Mills et $a l ., 2010)$. En este caso, el puma hembra adulto del segundo ambiente, «Yenni», realizaba este comportamiento el doble de tiempo que los otros tres pumas, lo cual podría indicar que esta conducta se estaba volviendo excesiva en ella.

\section{Conclusiones}

- La implementación del enriquecimiento ambiental en los pumas del Parque Zoológico Taraccasa fue positiva. Aumentaron los comportamientos deseables como la exploración, locomoción, búsqueda y juego.

- Conductas indeseables como el pacing, acicalamiento en exceso y descanso prolongado disminuyeron significativamente.

\section{Literatura Citada}

1. Beresca A. 2014. Enriquecimiento ambiental. En: Cubas Z (ed). Tratado de animais selvagens. Brazil: Roca. p 63-73.

2. Broom DM. 1988. The scientific assessment of animal welfare. Appl Anim Behav Sci 20: 5-19. doi: 10.1016/01681591(88)90122-0
3. Diaz N. 2001. El efecto del enriquecimiento ambiental sobre los niveles de actividad el uso del espacio y la interacción social en pumas (Felis concolor) y linces (Lynx rufus) albergados en el zoológico «Los Coyotes». Tesis Licenciatura. México: Univ Nacional Autónoma de México. $78 \mathrm{p}$.

4. Estrada G, Parra J. 2007. Enriquecimiento ambiental de fauna silvestre sometida a cautiverio en el hogar de Paso Uniamazonia - Corpoamazonia. Revista CES 2(2): 8-13.

5. Harmsen B, Foster R, Silver S, Ostro L, Doncaster CP. 2010. Jaguar and puma activity patterns in relation to their main prey. Mammalian Biol 76: 320-334. doi: 10.1016/j.mambio.2010.08.007

6. Manteca $X$. 2003. Etología clínica veterinaria del perro y gato. España: Multimédica. $261 \mathrm{p}$.

7. Martínez-Pulido C, Cortés-Corredor LR, Cepeda-Barragán LT, MuñozAgudelo LM, Soler-Tovar D, Mayor GM. 2014. Estudio preliminar del comportamiento de Puma concolor como indicador de bienestar en dos colecciones zoológicas de Cundinamarca. En: Mem Conf Interna Med Aprovech Fauna Silv Exót Conv. Colombia. p 11-24.

8. Mason G, Rushen J. 2006. Stereotypic animal behaviour: fundamentals and applications to welfare. $2^{\text {nd }}$ ed. Oxforshire, UK: CABI. 367 p.

9. Medrano G 2008. Implementación de un programa de enriquecimiento ambiental y sus efectos conductuales sobre un grupo de felinos (Panthera onca, Panthera leo, Panthera tigris altaica, Felis concolor) en cautiverio del Parque Zoológico Miguel Ángel de Quevedo. Tesis de Médico Veterinario Zootecnista. Veracruz, México: Univ Veracruzana. $99 \mathrm{p}$.

10. Mills DS, Marchant-Forde J, McGreevy P, Morton D, Nicol C, Phillips C, Sandoe P, Swaisgood R. 2010. The encyclopedia of applied animal behaviour and welfare. Oxforshire, UK: CABI. 704 p. 
11. Morgan K, Tromborg C. 2006. Sources of stress in captivity. Appl Anim Behav Sci 102: 262-302. doi: 10.1016/ j.applanim.2006.05.032

12. Newberry R. 1995. Environmental enrichment: increasing the biological relevance of captive environments. Appl Anim Behav Sci 44: 229-243. doi: 10.1016/0168-1591(95)00616-Z

13. Pacheco P. 2005. Enriquecimiento ambiental en jaguares (Pathera onca) en el Zoológico Zacango. Tesis de Licenciatura. México: Univ Nacional Autónoma de México. 79 p.
14. Rodríguez J, Alberico M, Trujillo F, Jorgenson J. 2006. Libro rojo de los mamíferos de Colombia. Bogotá, Colombia: Ministerio de Ambiente, Vivienda y Desarrollo Territorial. $430 \mathrm{p}$.

15. Skibiel A, Triveno H, Naugher K. 2007. Comparison of several types of enrichment for captive felids. Zoo Biol 26: 371-381. doi: 10.1002/zoo.20147

16. Villafuerte M. 2012. Estudio del comportamiento en cautividad del puma (Puma concolor) y jaguar (Panthera onca) en el parque zoológico «Benito Juarez». Tesis de Médico Veterinario Zootecnista. México: Univ Michoacana de San Nicolás de Hidalgo. 52 p. 